\title{
Ce que la génétique dit, la traduction le fait
}

\section{Fabienne Durand-Bogaert}

\section{OpenEdition}

\section{Journals}

Édition électronique

URL : http://journals.openedition.org/genesis/995

DOI : 10.4000/genesis.995

ISSN : 2268-1590

\section{Éditeur :}

Presses universitaires de Paris Sorbonne (PUPS), Société internationale de génétique artistique littéraire et scientifique (SIGALES)

\section{Édition imprimée}

Date de publication : 15 avril 2014

Pagination : 7-10

ISBN : 9782840509370

ISSN : 1167-5101

\section{Référence électronique}

Fabienne Durand-Bogaert, « Ce que la génétique dit, la traduction le fait », Genesis [En ligne], 38 | 2014 , mis en ligne le 29 juin 2016, consulté le 22 septembre 2020. URL : http://journals.openedition.org/ genesis/995; DOI : https://doi.org/10.4000/genesis.995 


\section{Ce que la génétique dit, la traduction le fait}

$E$ Itre la génétique des textes et cette catégorie particulière d'écrits que sont les traductions, la rencontre était prévisible : inscrite dans ce qui anime, au sens propre, l'esprit et le faire de l'une et de l'autre. La génétique enquête sur la fabrique, débusque les processus, remonte le cours de l' œuvre, scrute les traces. La traduction est fabrique - de langues, de pensée, de textes, de littératures dites étrangères (mais qui, grâce à elle, le sont un peu moins). Elle est processus, mise en mouvement perpétuelle, vouée d'avance à la reconduction. Traduire, en effet, c'est transporter un texte d' une langue et d' une culture vers d' autres tout en sachant l'arrimage provisoire (sauf cas d'infatuation extrême, aucun traducteur ne fera jamais voisiner son nom avec la mention «traduction définitive »). Enfin, la traduction, en refaisant pas à pas le chemin parcouru par l'auteur, est doublement trace : de ce parcours à rebours, dont l'indice matériel est la production d'un discours - mots, sonorités, rythmes, voix - à travers lequel se profile l'empreinte d'une lecture, et parfois d'une interprétation. Les procédures de la génétique, la traduction les met en cuvre à tout instant : son être même est déjà performatif.

Entre Genesis et la traduction, la rencontre est aussi plus tardive, comparativement à d'autres domaines de la création - philosophie, cinéma, arts plastiques, musique, entre autres - qui ont tôt éveillé l'intérêt des généticiens. Cela s'explique en partie par le fait que les recherches sur la traduction, longtemps confinées à la périphérie de disciplines telles que la littérature comparée, la linguistique ou la stylistique, ont d'abord eu la tâche de conquérir leur autonomie. En France, on peut dater le début de l'émancipation des premiers travaux de Henri Meschonnic sur le rythme1 (1982), bientôt suivis de la réflexion d'Antoine Berman sur la traduction dans l'Allemagne romantique 2 (1984). Le premier souci de ces pionniers fut de poser les bases d'une critique des traductions qui ne soit ni de jugement - esthétique ou moral - ni de sensibilité, mais s'attache à dégager les modalités selon lesquelles une traduction, en remontant à l'intention ou visée originale d'une æeuvre, en potentialise le « sens infini » $F$. Schlegel). Tant d'idées reçues étaient à secouer, héritées d'une conception très négative de la traduction comme prestation de service, produit dérivé, succédané approximatif de l'original qu'il n'est guère surprenant que la critique des traductions ne se soit édifiée, à ses débuts, que sur la base des textes imprimés. Sans doute aussi, toute réflexion sur le traduire est-elle toujours hantée par le souvenir de l'étroite solidarité qui unit historiquement traduction, exégèse et herméneutique. Or cette solidarité passant essentiellement par la philologie, c'est d'avec les procédures de cette

1. Henri Meschonnic, Critique du rythme, Paris, Verdier, 1982.

2. Antoine Berman, L'Épreuve de l'étranger. Culture et traduction dans l'Allemagne romantique, Paris, Gallimard, coll. « Les Essais », 1984. 
dernière que la critique des traductions devait d'abord se démarquer. À cet égard, la publication, en 1995, de Pour une critique des traductions : John Donne d'Antoine Berman dissipa toute possible et malencontreuse confusion.

Presque vingt ans plus tard, nous sommes parvenus à un tournant dans les recherches sur la traduction. Les avancées sont nombreuses et stimulantes dans le champ de l'histoire des traductions, inséparable de celui de leur critique. Une traduction étant toujours faite dans l'intérêt de la culture d'accueil, les chercheurs se sont notamment appliqués à une recontextualisation historique et culturelle des traductions, s'efforçant de déterminer les liens que telle ou telle traduction entretient avec la poétique dominante de l'époque et du pays où elle voit le jour. Sans cet effort de recontextualisation, en effet, le risque est grand de ne lire les textes traduits qu'à la lumière de critères par trop contemporains. Porter à l'encontre d'une traduction l'accusation d' "ethnocentrisme ", par exemple, peut valoir dans la perspective actuelle, mais être dénué de sens à la fin du XIXe siècle français ou dans l'Angleterre des années 1920. Car non seulement les significations changent, mais les mots s'usent, la langue bouge, se renouvelle, et comme l'avait pressenti Walter Benjamin, "pendant que la parole de l'écrivain perdure dans ce qui lui est propre, c'est le destin même de la plus grande traduction de disparaître dans la croissance de sa langue ${ }^{3}$ »-d'être à refaire, donc. D'autre part, chaque époque possède sa propre doxa traduisante par rapport à laquelle toute traduction doit pouvoir être située, qu'elle s'y conforme ou s'en démarque ostensiblement.

À côté de cette "macro-recontextualisation " dont la cause est désormais entendue, il existe une recontextualisation plus fine, plus ciblée que seule l'approche génétique permet d'envisager ${ }^{4}$. Les conditions précises dans lesquelles une traduction prend forme n'ont guère encore été étudiées : l'atelier du traducteur reste un mystère, comme s'il s'y fomentait quelque entreprise dont seul le résultat - la traduction publiée - importait, le processus effaçant ses traces à mesure. Car ce n'est pas le moindre des paradoxes : alors que les traducteurs s'indignent volontiers (et avec juste raison) du caractère arbitraire des révisions auxquelles le processus éditorial soumet leur texte, la plupart d'entre eux ne jugent pas utile de conserver les traces des différentes campagnes qui leur ont permis d'aboutir à tel ou tel choix lexical ou syntaxique. Ils tarissent ainsi eux-mêmes la source à laquelle ils pourraient puiser de précieux arguments à l'appui d' une cohérence traduisante dont la mémoire humaine n'a pas toujours le souvenir.

Mais si les traducteurs effacent souvent leurs traces, la raison en est aussi à chercher $d u$ côté d'une auctoritas fragilisée par des siècles de réticences à penser la traduction comme activité de création - et non de simple recréation. L'article intitulé "Les deux corps du texte », qui ouvre la section "Enjeux », détaille les raisons de cette marginalisation de la traduction dans l'espace des productions textuelles, dont l'une des conséquences directes est la rareté des brouillons, ou à tout le moins leur caractère parcellaire. Cette donnée, qui complique certes un peu la tâche du généticien, ne la rend cependant pas impossible. Elle l'incite seulement à exploiter la richesse d'un autre matériau, que l'on dira, selon les cas, avant-textuel ou paratextuel : préfaces et carnets de travail du traducteur, correspondances entre le traducteur et l'auteur ou l'éditeur, autres productions textuelles du traducteur

3. Walter Benjamin, « La tâche du traducteur », trad. Martine Broda, Po\&sie, n 55, 1991, p. 153.

4. Dès 2011, la revue brésilienne Manuscritica a consacré son numéro 20 à la genèse de la traduction. 
(adaptations, articles de presse ou scientifiques), etc. Le second article de la section "Enjeux » constitue au reste la démonstration magistrale du profit que le généticien peut tirer de ce matériau : une traduction étant toujours le produit d'une lecture, c'est à l'analyse des annotations portées par Elmar Tophoven sur son exemplaire de La Jalousie d'Alain Robbe-Grillet et sur le volume allemand correspondant que nous convie Jean-Louis Lebrave. À travers ces annotations se révèlent non seulement la façon dont le traducteur a circulé entre les données du texte français, mais aussi la manière dont il les a organisées, voire recomposées dans le passage à l'autre langue - l'allemand-, le lent tissage d'un autre texte.

Les cinq "Études » qui suivent illustrent quelques-unes des pistes de recherche proposées dans la rubrique "Enjeux». Un premier ensemble prend appui sur le corpus joycien. Marie-Hélène Paret-Passos présente les carnets de travail du traducteur brésilien de Finnegans Wake, Donaldo Schüler - soit trois cahiers de commentaires et de notes de lecture préparatoires et dix cahiers consignant un premier jet de traduction. L'exploration de ce matériau donne une assise matérielle aux concepts de "position traductive » et de "projet de traduction " énoncés par Antoine Berman, dont elle confirme l'intérêt pour l'approche génétique. Tiphaine Samoyault, qui a collaboré à la retraduction de Ulysses (Gallimard, 2004), renverse le point de vue qui prévaut habituellement en envisageant la traduction comme le brouillon postérieur de l'œuvre, reformulant ainsi la notion de pluriel de l'auvre : loin d'être la réduction du multiple des possibles à l'un, la traduction est au contraire le point de départ de son extension infinie.

C'est bien aussi de la potentialisation de l' cuvre à travers la traduction comme fable ou comme pratique qu'il est question dans la mise en regard qu'opère Chiara Montini du roman Le Désert mauve (1987) de Nicole Brossard, et de trois versions (française, anglaise et italienne) de Mercier et/and/e Camier de Samuel Beckett. Ici, genèse et traduction s'entrecroisent en un jeu de relance et d'engendrement perpétuel, dont l'autotraduction est l'un des principaux moteurs. En effet, l'autotraduction, dont la pratique résulte le plus souvent du bilinguisme des auteurs - quand ce n'est pas de leur trilinguisme, comme chez Vladimir Nabokov auquel Olga Anokhina consacre son étude - pose à la traduction comme à l'approche génétique des questions passionnantes : à un seul et unique "auteur» correspondent non seulement des versions plurilingues d'un "même » (mais l'est-il ?) texte, mais aussi une posture complexe. Ne s'autorisant que de lui-même - jusque dans l'autocensure -, l'auteur-traducteur se campe souvent en adversaire redoutable de tout traducteur «allogène ». Olga Anokhina détaille, à cet égard, les diverses stratégies mises en auvre par Nabokov, qui supervisait d'un oeil sévère le travail de ses traducteurs, traitant volontiers leur texte comme une simple toile de fond.

La réflexion sur le traduire présente, par ailleurs, une particularité qui nous a semblé devoir être soulignée : c'est l'un des domaines - la musicologie en est un autre - dans lequel la plupart des chercheurs sont aussi des praticiens. Daria Sinichkina a participé, entre avril et juin 2012, à l'atelier franco-russe de traduction organisé par le CITL (Collège international des traducteurs littéraires d'Arles). Elle analyse ici la genèse de sa propre traduction d'un chapitre de L'Arabesque d'or (1925), le roman de Boris Zaïtsev, montrant le passage qui s'effectue de l'altérité du texte étranger à son apprivoisement, du foisonnement et de l'éclatement des significations dans les brouillons à la patiente construction d' une cohérence traductive. 
Que la traduction ait aussi valeur heuristique, les deux «Entretiens » qui composent la rubrique éponyme en témoignent. Barbara Cassin revient sur la genèse du Vocabulaire européen des philosophies (2004), montrant que la discordance des mots est au principe même de la fabrique de la pensée, et précise les prolongements présents et à venir notamment sous forme hypertextuelle - de son entreprise. Sandrine Marchand, quant à elle, nous transporte en Orient, dans l'univers de l'écrivain tä̈wanais Wang Wen-hsing, qu'elle traduit. La traduction est ici le lieu d'une double épreuve de l'étranger : Sandrine Marchand s'y confronte non seulement à l'hétérogénéité des langues - l'auteur de Processus familial et Un homme dos à la mer invente son propre idiome plurilingue -, mais elle éprouve aussi l' effet-retour de la traduction sur sa propre écriture poétique. S' insinuant dans son français comme par un phénomène de contamination, le chinois lui rend sa propre langue étrangère.

Enfin, dans le prolongement de l'entretien avec Sandrine Marchand, nous présentons un document inédit : le dossier génétique constitué par les brouillons successifs de la traduction de Récit impromptu de mon retour à la maison de Yuan Mei, l'un des plus grands poètes chinois du XVIII siècle.

Fabienne Durand-Bogaert

\section{Varia}

Nous retrouvons Wang Wen-hsing dans la première étude de la rubrique "Varia 》. Genesis est heureux de présenter pour la première fois une étude de genèse portant sur une écriture non occidentale. Même si les processus d'écriture très particuliers du romancier d'avant-garde tä̈wanais ne peuvent être considérés comme représentatifs de l'écriture idéogrammatique en général, c'est tout un champ nouveau qui s'ouvre à la critique génétique, dans lequel il faudra trouver de nouveaux repères. L'article de Peng Yi permet notamment d'observer l'importance des blancs, de la ponctuation et des signes ayant trait à l'intonation. Avec l'étude suivante de Danielle Terrien et Jean-Jacques Queloz, nous ne quittons pas vraiment la Chine, ou du moins l'inspiration poétique chinoise, puisqu'il s'agit d' un poème de Victor Segalen, tiré de Stèles : "Trahison fidèle ». L'examen des sept versions du poème permet de suivre son élaboration et met en évidence le dialogisme de sa composition.

La section «Chroniques » comporte, comme tous les numéros pairs, une bibliographie des travaux récents. On y trouve aussi les comptes rendus de deux ouvrages importants à des titres divers : l'édition historique des fragments autographes de l'Orlando Furioso par Santorre Debenedetti, republiée avec une préface de Cesare Segre, et l'étude transversale des dossiers génétiques de Zola, proposée par Olivier Lumbroso, dont Henri Mitterand souligne ici le caractère novateur.

La Rédaction de Genesis 